Original Article

\title{
Early Postoperative Outcome of Off-Pump Coronary Artery Bypass Grafting: A Report from the Highest-Volume Center in Japan
}

\author{
Kishio Kuroda, MD, ${ }^{1}$ Tomoko S. Kato, MD, PhD, ${ }^{1}$ Kenji Kuwaki, MD, PhD, ${ }^{1}$ \\ Kan Kajimoto, MD, PhD, ${ }^{1}$ Seitetsu L. Lee, MD, ${ }^{2}$ Taira Yamamoto, MD, PhD, ${ }^{1}$ \\ and Atsushi Amano, $\mathrm{MD}, \mathrm{PhD}^{1}$
}

\begin{abstract}
Background: Off-pump coronary artery bypass grafting (OPCAB) has evolved into a standard technique in coronary artery bypass grafting. However, a detailed investigation in Japanese population undergoing OPCAB has not yet been fully elucidated.

Methods: A total of 1109 consecutive patients undergoing isolated OPCAB between 2006 and 2013 at Juntendo University were reviewed. The data was evaluated in the light of previously published $\mathrm{OPCAB}$-associated reports.

Results: There were 904 male $(81.5 \%)$ and a mean was $67.5 \pm 9.8$ years. Eight patients $(0.5 \%)$ died within 30 days postoperatively or before discharge, which was equivalent to or rather better than the previously reported mortality rates, including the European System for Cardiac Operation Risk Evaluation II (2.1 \pm 2.1) data. A morbidity analysis revealed that prolonged intubation ( $>24 \mathrm{~h}$ ) occurred in 43 patients $(3.6 \%)$, surgical site infections in $18(1.9 \%)$, neurological complications in $13(1.3 \%)$. A reduced preoperative left ventricular ejection fraction (less than $\mathbf{4 0 \%}$ ) was found to be a risk factor for early postoperative death (odds ratio 10.58 , respectively, $p<0.05$ ) in our cohort.

Conclusions: Early postoperative mortality and morbidity rates in Japanese population after OPCAB were rather satisfactory and similar to those reported by other countries.
\end{abstract}

Keywords: off-pump coronary artery bypass grafting, Japanese population, outcome

\section{Introduction}

Ischemic heart disease (IHD) is a major cause of death worldwide, and is responsible for about one-third or more of all deaths in individuals older than 35 years. ${ }^{1,2}$

${ }^{1}$ Department of Cardiovascular Surgery, Juntendo University School of Medicine, Tokyo, Japan

${ }^{2}$ Department of Cardiovascular Medicine, The University of Tokyo, Tokyo, Japan

Received: May 17, 2015; Accepted: October 22, 2015 Corresponding author: Tomoko S. Kato, MD, PhD. Heart Center, Department of Cardiovascular Surgery, Juntendo University, 2-1-1 Hongo, Bunkyo-ku, Tokyo 113-8421, Japan Emails: tokato@juntendo.ac.jp, rinnko@sannet.ne.jp (C)2015 The Editorial Committee of Annals of Thoracic and Cardiovascular Surgery. All rights reserved.
Coronary artery bypass grafting (CABG) is a standard treatment option for patients with coronary artery disease. The indication for CABG is considered to be dependent on multiple factors. ${ }^{3)}$ In particular, CABG is generally preferred for patients with relatively broad lesions at risk of ischemia, with an unprotected left main trunk or/and diffuse three-vessel disease. According to an annual report by the Japanese Association for Thoracic Surgery, more than $15000 \mathrm{CABG}$ procedures have been performed over the past few years in Japan. ${ }^{4)}$ CABG is usually performed with the use of cardiopulmonary bypass $(\mathrm{CPB})$, the so-called on-pump beating $\mathrm{CABG}$ (ONCAB). The use of $\mathrm{CPB}$ requires cannulation to the aorta, which may increase the risk of stroke especially in patients with calcified or atherosclerotic ascending aorta. Furthermore, CPB may cause hemodynamic alteration 


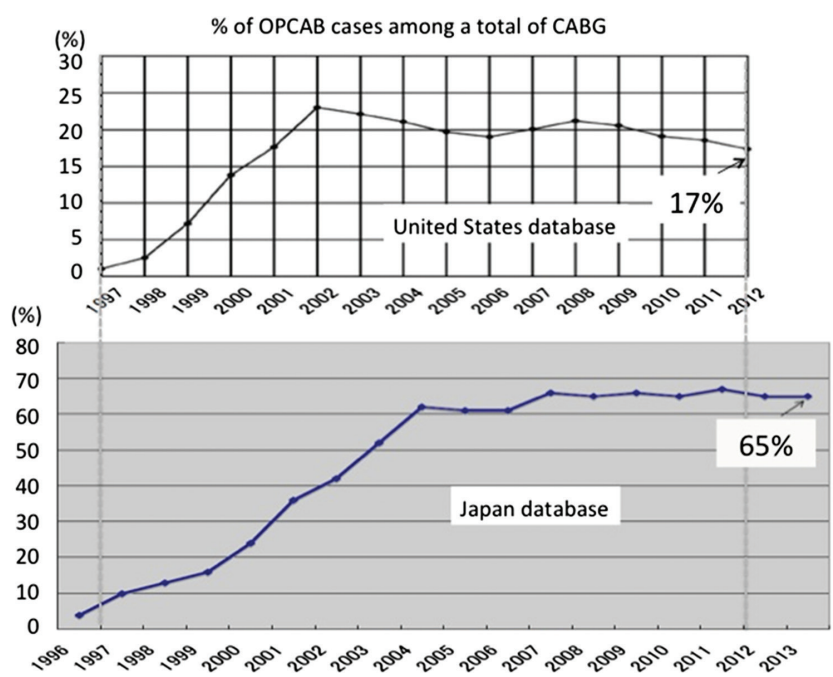

Fig. 1 The changes in a proportion of OPCAB in total CABG cases in the US (above) and in Japan (below) by era. The data was obtained from reports in the Japanese Associate for Coronary Artery Surgery) and the Society of Thoracic Surgeons Adult Cardiac Surgery, ${ }^{9}$ ) and reconstructed. The OPCAB case in 2012 in the US was about $17 \%$, whereas it was $65 \%$ in 2013 in Japan. OPCAB: off-pump coronary artery bypass grafting; CABG: coronary artery bypass grafting

and cytokine release. ${ }^{5)}$ In contrast, off-pump (i.e., without $\mathrm{CPB}$ on the beating heart) coronary artery bypass (OPCAB) prevents such adverse effects associated with $\mathrm{CPB}$, although it should be performed by an experienced surgeon. According to the multiple advantages of $\mathrm{OPCAB}$ over ONCAB in addition to the recent advances in surgical devices such as the stabilization system of targeted vessels, OPCAB has evolved into a standard technique. $\left.{ }^{6}\right)$ Especially in Japan, surgeons have become proficient in OPCAB techniques in the past 10 years. Therefore, a proportion of OPCAB among total CABG cases in Japan has reached more than $60 \%,{ }^{4,7,8)}$ which is extremely higher than that in the US (17\%) (Fig. 1). ${ }^{4,9)}$ Although long-term prognosis in patients who underwent OPCAB is controversial, this technique has been reported to reduce the occurrence of perioperative complications. ${ }^{10)}$ However, the advantages of OPCAB over ONCAB have not been fully clarified, because the number of cases reported by each center that participated in OPCAB-related studies varies a lot to draw conclusions. ${ }^{11)}$ Indeed, only a few studies on OPCAB performed in Japan have been reported. Of note, our institution has actively performed $\mathrm{OPCAB}$ in a routine manner, and tends to use $\mathrm{CPB}$ only in limited cases these days (Fig. 2). Therefore, in the present report, we reviewed the clinical characteristics

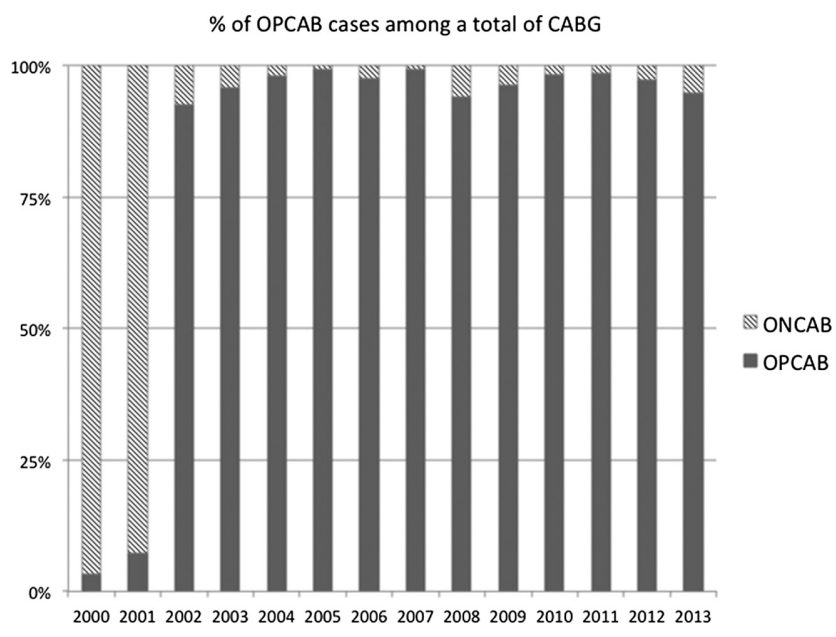

Fig. 2 The changes in a proportion of OPCAB in a total CABG cases at our institution. The OPCAB cases exceed $90 \%$ of total CABG cases since 2002. OPCAB: off-pump coronary artery bypass grafting; CABG: coronary artery bypass grafting

and early postoperative outcomes of patients who underwent $\mathrm{OPCAB}$ at our institution, which is one of the high-volume centers in Japan, and evaluated the results in the light of previously published OPCAB-related papers.

\section{Material and Methods}

\section{Patients}

At Juntendo University Hospital in Japan, 1203 consecutive patients who underwent elective isolated OPCAB between December 2006 and October 2013 were reviewed. Of these, 94 patients who underwent an emergency procedure, re-sternotomy operation, and conversion to extracorporeal circulation-supported surgery were excluded from the study. The remaining 1109 patients were included in the present analysis.

\section{Study design}

We summarized the preoperative and postoperative courses in our cohorts. Postoperative death was defined as death occurring within 30 days after surgery or during hospitalization. Neurological complication was defined as a focal or global neurological deficit including transient ischemic attack (TIA) that resolves within $24 \mathrm{~h}$, and ischemic or hemorrhagic intracranial stroke persisting beyond $24 \mathrm{~h}$ accompanied by an imaging study. Renal dysfunction is defined by the presence of organic kidney damage or a persistent decrease in estimated glomerular filtration rate (eGFR) $<60 \mathrm{~mL} / \mathrm{min} / 1.73 \mathrm{~m}^{2}$ (Chronic kidney damage stage $3 \mathrm{a}$ or more severe) for three or more 
months. Diabetes was defined as HbA1c $\geq 6.5 \%$ [NGSP] or a status requiring antidiabetic agents/insulin. Extracardiac arteriopathy was defined as claudication, carotid occlusion or as $>50 \%$ stenosis, amputation for arterial disease, or a history of intervention on the abdominal aorta, limb arteries or carotids. Respiratory complications were defined as indications for mechanical ventilation for longer than $24 \mathrm{~h}$ or development of pneumonia. Re-sternotomy was performed in patients who developed sudden massive bleeding or showed signs of cardiac tamponade. The data collection protocol was approved by the institutional review board of Juntendo University. Our data was evaluated in the light of previously published reports, which focused on early postoperative mortality and morbidity following OPCAB..$^{7,12-14)}$

\section{Surgical techniques}

All the procedures were performed through a median sternotomy. The conduits (one or both internal thoracic arteries, the right gastroepiploic artery, and the saphenous vein) were harvested and skeletonized. Arterial conduits were used whenever possible. The arterial grafts are covered with warm papaverine hydrochloride gauze, and spasms are released with olprinone injections. The left side pericardium retracted sutures and two deep pericardial sutures are placed so as to position the apex towards the ceiling. These procedures and a suction stabilizer enable us to approach any region. A suction stabilizer is used to fix in place the coronary artery site for anastomosis, and the coronary artery is exposed. In patients with a risk of hemodynamic instability, CPB usage was considered appropriate. An incision is made in the coronary artery, and the central side is snared with an elastic string to create a bloodless operative field. Internal shunt tubes are used if there is excessive coronary blood flow and if hemorrhage or myocardial ischemia is a concern. Carbon dioxide (which is water-soluble and not prone to causing embolisms) is blown onto the site with a $\mathrm{CO}_{2}$ blower to locally disperse blood and to assist in achieving a bloodless operative field. Coronary artery anastomosis was performed by using continuous $8-0$ or 7-0 propylene sutures. For proximal anastomosis with free grafts of the ascending aorta, systemic blood pressure was maintained at around $80 \mathrm{mmHg}$, and anastomosis was performed with a continuous 6-0 propylene suture on a partially clamped aorta. If clamping of the ascending aorta was not possible due to calcification or plaque, an automatic anastomotic device was used. After completion of all anastomoses, a transit time flow measurement device was used
Table 1 Patients' characteristics

\begin{tabular}{|c|c|}
\hline Variable & $\mathrm{n}=1109$ \\
\hline Age, years & $67.5 \pm 9.8$ \\
\hline Sex male, $\mathrm{n}(\%)$ & $904(81 \%)$ \\
\hline Body mass index, $\mathrm{kg} / \mathrm{m}^{2}$ & $23.6(21.6-25.8)$ \\
\hline Diabetic mellitus, n (\%) & $633(57 \%)$ \\
\hline Dyslipidemia, n (\%) & $316(28 \%)$ \\
\hline Hypertension, n (\%) & $859(77 \%)$ \\
\hline Chronic kidney disease, n (\%) & $440(39 \%)$ \\
\hline Hemodialysis, $\mathrm{n}(\%)$ & $80(7 \%)$ \\
\hline $\begin{array}{l}\text { Neurological complications, } \\
\mathrm{n}(\%)\end{array}$ & $177(16 \%)$ \\
\hline Arteriopathy, n (\%) & $159(14 \%)$ \\
\hline COPD, n $(\%)$ & $120(11 \%)$ \\
\hline Hemoglobin, g/dL & $12.7 \pm 1.7$ \\
\hline $\begin{array}{l}\text { Brain natriuretic peptide, } \\
\mathrm{pg} / \mathrm{mL}\end{array}$ & $172.2 \pm 420.6$ \\
\hline $\mathrm{HbA} 1 \mathrm{c}, \%$ & $5.9(5.4-6.7)$ \\
\hline Total cholesterol, mg/dL & $165.0(142.0-190.8)$ \\
\hline Triglyceride, mg/dL & $115.0(84.0-159.8)$ \\
\hline $\begin{array}{l}\text { Low density lipoprotein, } \\
\mathrm{mg} / \mathrm{dL}\end{array}$ & $94.0(75.0-116.0)$ \\
\hline $\begin{array}{l}\text { High density lipoprotein, } \\
\text { mg/dL }\end{array}$ & $43.0(36.0-52.0)$ \\
\hline $\mathrm{eGFR}, \mathrm{mL} / \mathrm{min} / 1.73 \mathrm{~m}^{2}$ & $65.4(49.1-79.7)$ \\
\hline Creatinine, $\mathrm{mg} / \mathrm{dL}$ & $0.8(0.7-1.1)$ \\
\hline LVEF, \% & $59.0(48.0-66.0)$ \\
\hline Number of diseased vessels, $n$ & $3.1 \pm 0.8$ \\
\hline NYHA classification & $1.2 \pm 1.1$ \\
\hline CCS classification & $2.0 \pm 0.5$ \\
\hline EuroSCORE II, \% & $1.4(1.0-2.3)$ \\
\hline
\end{tabular}

COPD: chronic obstructive pulmonary disease; eGFR: estimated glomerular filtration rate; LVEF: left ventricular ejection fraction measured by echocardiographic analysis; NYHA: New York Heart Association Functional Classification; CCS: Canadian Cardiovascular Society Functional Classification

to ensure sufficient blood flow in all the grafts, and the bypass was completed. ${ }^{15)}$ In order to prevent postoperative arrhythmia-induced cardiac emboli, left atrial appendage amputation and/or occlusion (LAA A/C) has been performed in our institution since 2012. This is because the LAA is the main source of emboli in patients with atrial fibrillation (Af), which is the most prevalent arrhythmia after cardiac surgery. The LAA procedure is performed with a purse-string suture and a continuous over and over suture. ${ }^{16)}$

\section{Statistical analysis}

Data were presented as mean \pm SD values for continuous variables and as percentages for categorical variables. Normality was evaluated for each variable from normal 
distribution plots and histograms. Where data were not normally distributed, the median value associated with the interquartile range is presented. Factors associated with postoperative outcome were evaluated by univariate and multivariate logistic regression analyses based on the available clinical data. For the purpose of including variables into uni- and multivariate logistic regression analysis, data that showed a bimodal distribution were subjected to logarithmic transformation of the variables as needed to improve normality before performing the statistical analysis. Co-variables which were found to be associated with combined morbidities by univariate analysis $(p<0.05)$ were entered into the subsequent multivariate analysis. Statistical analyses were performed using the Statistical Analysis System Software JMP 11.0 (SAS Institute Inc. Cary, NC, USA).

\section{Results}

The present observation included 1109 patients who underwent $\mathrm{OPCAB}$ during the study period. Their preoperative clinical characteristics are shown in Table 1. Of these patients, 904 were men (81.5\%), 859 (77.2\%) had a history of hypertension, and 633 (57.3\%) had diabetes mellitus. In addition, the European system for cardiac operative risk evaluation showed that the estimated mortality within 30 days after cardiac surgery in the present cohort was $2.1 \pm 2.1$. Among all patients, 186 (16.7\%) underwent simultaneous LAA A/C at the time of OPCAB. The effect of the LAA procedure on their postoperative outcomes is now under investigation (data not shown).

\section{Perioperative mortality and morbidity}

Table 2 shows the perioperative mortality and morbidity. The overall mortality rate was $0.5 \%$ in this cohort. Around $30 \%$ of the patients developed postoperative arrhythmia, most of whom had Af. Forty-three patients (3.6\%) had respiratory complications, 32 (3.3\%) had newly developed renal failure that required renal replacement therapy of whom two $(0.2 \%$ in total cohort) required permanent hemodialysis, and $13(1.3 \%)$ had postoperative neurological complications,

The detailed information about patients who died during hospitalization is summarized in Table 3. Among eight deaths, there were two cardiovascular deaths and six non-cardiac deaths. Half of the patients who died were already on chronic hemodialysis prior to the surgery, and one patient required transient renal replacement therapy postoperatively. Six out of eight patients developed cardiac
Table 2 Peri- and postoperative data

$\begin{array}{lc}\text { Variable } & \mathrm{n}=1109 \\ \text { Operation time, min } & 278.0(252.0-328.0) \\ \text { Number of grafts, } \mathrm{n} & 3.5 \pm 1.3 \\ \text { Mortality, n (\%) } & 8(0.5 \%) \\ \text { Neurological complications, } \mathrm{n}(\%) & 13(1 \%) \\ \text { Respiratory complications, } \mathrm{n}(\%) & 43(4 \%) \\ \text { GI bleedings, n (\%) } & 2(0.1 \%) \\ \text { Surgical site infection, n (\%) } & 18(2 \%) \\ \text { ICU stay, days } & 1(1-1) \\ \text { Postoperative hospital stay, days } & 9(8-11)\end{array}$

GI: gastrointestinal; ICU: intensive care unit

arrhythmia. The mean duration from surgery to death was 6.5 (2-35) days.

\section{Outcome analysis of our data and those in the recent literatures}

Table 4 summarizes the outcomes of OPCAB reported in the literature ${ }^{7,12-14)}$ as referrals for our results. Of note, our observation was based on a single-center retrospective analysis, but the number of participants was sufficient. Triple-vessel bypass grafting surgery seemed to be less frequently performed under OPCAB in general, according to the literature. In the present investigation in our institution, the mean number of grafts was $3.5 \pm 1.3$. Peterson et al. performed an observational study in 267089 patients who underwent an isolated CABG procedure, not focusing on on-or off-pump procedure, and reported that the early postoperative mortality after $\mathrm{CABG}$ ranged from $2 \%$ to $5 \% .{ }^{17)}$ The mortality rates in the literature, which are shown in Table 4, also ranged from $1 \%$ to $6 \%$. Therefore, the mortality rate of $0.5 \%$ at our institution seemed to be satisfactory. The incidences of adverse events in our cohort other than new-onset Af, such as stroke or respiratory failure, were compatible with those reported in previous studies.

\section{Details of the patients who underwent ONCAB instead of OPCAB}

During the same period that the patients evaluated in the present study underwent OPCAB at our institution, only 14 patients underwent elective or urgent $\mathrm{ONCAB}$ as a conversion from OPCAB. The detailed data are summarized in Table 5. The mean age of this group was $68.4 \pm 12.0$ years, which was similar to that of the patients who underwent OPCAB. This group of patients had relatively impaired left ventricular function as reflected by preoperative left 


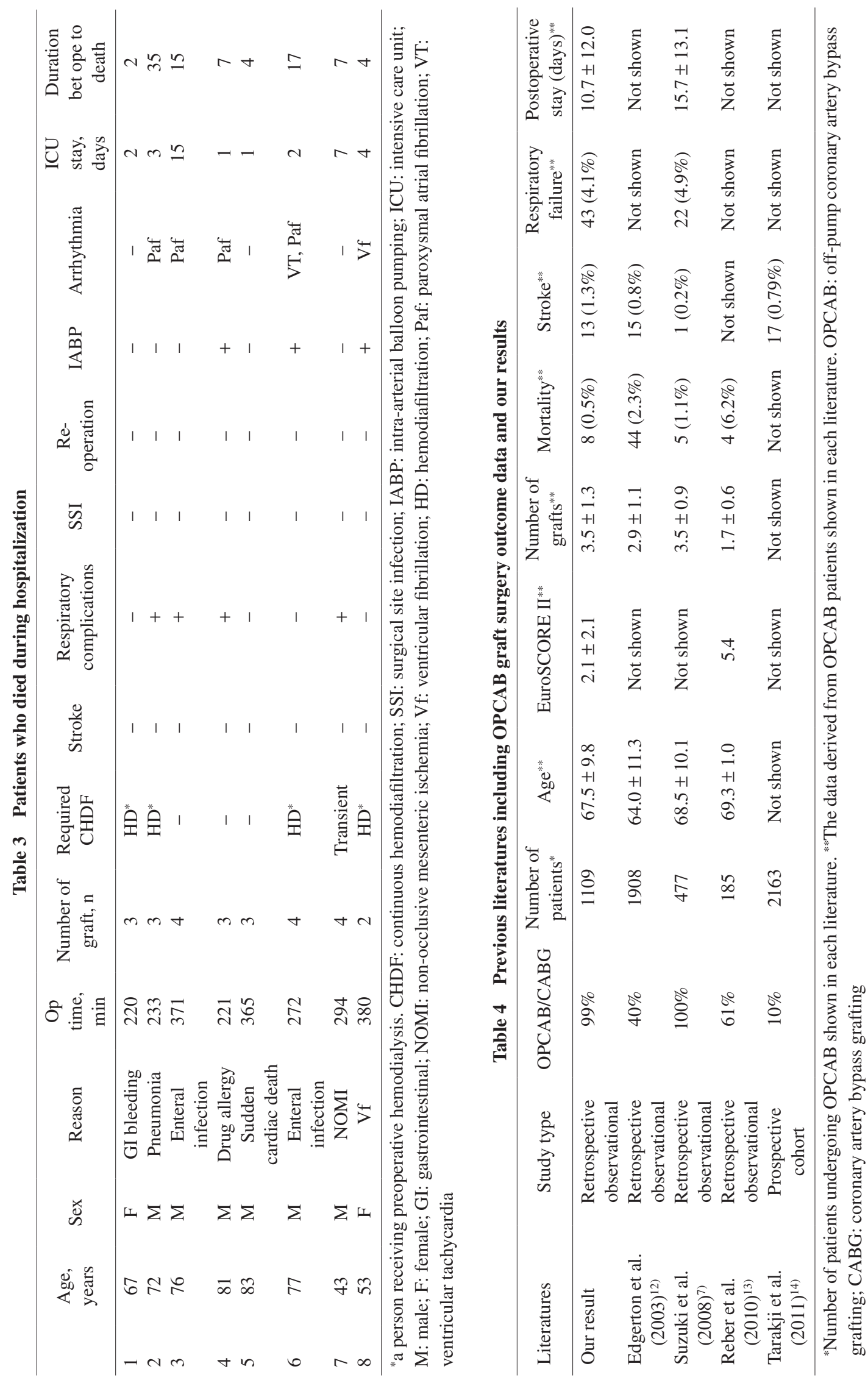




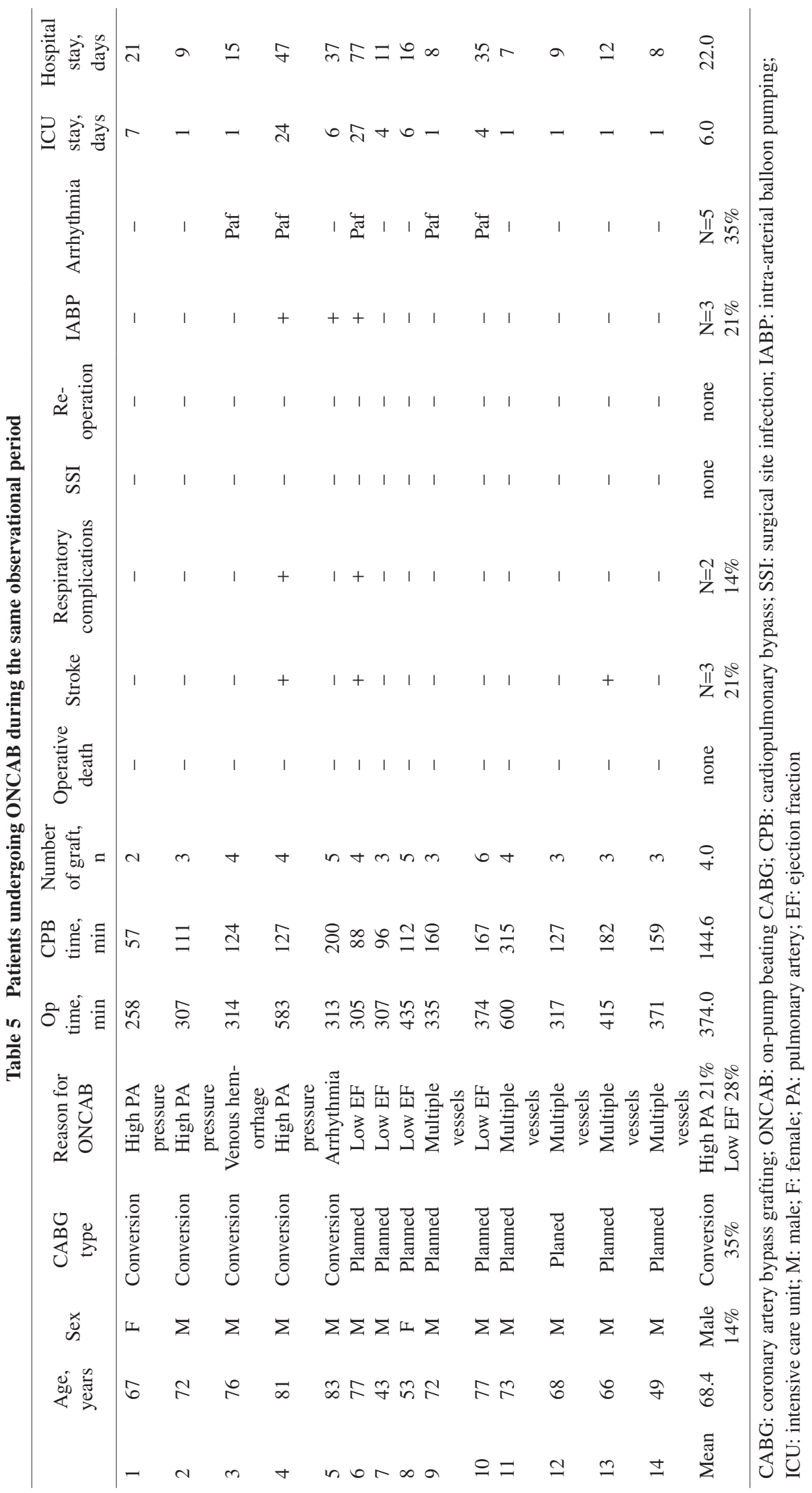


ventricular ejection fraction (LVEF) and higher predicted mortality derived from the EuroSCORE II. In our institution, we electively perform $\mathrm{ONCAB}$ rather than $\mathrm{OPCAB}$ in patients with impaired left ventricular function and multiplevessel disease because such patients are at risk of perioperative hemodynamic change, especially when anastomosis to the left circumflex coronary artery is planned. Furthermore, unexpected conversion from $\mathrm{OPCAB}$ to $\mathrm{ONCAB}$ is performed in patients whose pulmonary artery (PA) pressure has increased beyond the normal range under the procedure of apical elevation.

\section{Factors associated with mortality and morbidity based on an available dataset}

The event rates of both death $(n=8,0.5 \%)$ and combined morbidity $(n=85,8.7 \%)$ were not sufficient enough to perform analysis for preoperative determinants of mortality/morbidities. Furthermore, we could not obtain complete preoperative information from all patients. Therefore, although we admit that the analyses were limited to a crude conception derived from only available data, we performed univariate and multivariate logistic analyses for outcomes (Table 6). Among the variables we studied, reduced LVEF $(<40 \%)$, preoperative hemodialysis requirement, low body mass index (BMI), anemia, low high density lipoprotein (HDL), elevated creatinine concentration, severe preoperative conditions as reflected by a high CCS/EuroSCORE, and elevated brain natriuretic peptide (BNP) were found to be associated with postoperative mortality. For combined morbidities, advanced age, history of arteriopathy and stroke, reduced LVEF $(<40 \%)$, anemia, elevated creatinine concentration, reduced eGFR, high EuroSCORE and elevated BNP were found by univariate analysis to be risk factors. Due to the correlation between eGFR and creatinine concentration, eGFR, but not creatinine level, was entered into the multivariate analysis. The EuroSore consisted of several known risk factors, which were therefore not included in the multivariate analysis either. According to the multivariate analysis, previous stroke, reduced eGFR and elevated BNP levels remained as risk factors for combined morbidity.

\section{Discussion}

In the present report, we demonstrated that (i) the early postoperative mortality of the 1109 consecutive Japanese patients who underwent $\mathrm{OPCAB}$ at our institution was $0.5 \%$, which was much lower than the estimated mortality as calculated based on EuroSCORE II of 2.1; (ii) early postoperative adverse events, including stroke and respiratory failure, occurred in approximately $9 \%$ of the patients, which was equivalent to the morbidity incidences shown in previous literatures from the US or Europe; (iii) the ratio of ONCAB to OPCAB during the study period was 14:1109 (0.01) at our institution; (iv) patients with impaired left ventricular function and pulmonary hypertension tended to receive elective ONCAB; and (v) preoperative reduced LVEF may predict a poor outcome.

According to the 2013 investigation in Japan, initial elective OPCAB was performed in 5830 patients, and the mortality was $\left.0.82 \%{ }^{4}\right)$ Edgerton et al. reported a mortality rate of $2.3 \%$ in 1908 patients who underwent OPCAB in their retrospective study. ${ }^{12)}$ In the largest multicenter randomized trial to date that compared OPCAB with ONCAB (2203 patients), Shroyer et al. reported that the 30-day mortality rate was $2.5 \% .{ }^{18}$ ) Considering the estimated mortality rate of our patients that was derived from EuroSCORE II and by referring to the mortality rates reported in the literatures shown in Table 3, ${ }^{7,12-14)}$ we assume that the outcome of OPCAB at our institution was satisfactory.

Several studies reported that OPCAB is a useful technique for reducing postoperative mortality and morbidity compared with ONCAB. ${ }^{19,20)}$ Therefore, several institutions actively perform $\mathrm{OPCAB}$ rather than $\mathrm{ONCAB}$, especially in patients at high risk for postoperative complications, considering its beneficial effect of avoiding CPB. ${ }^{21)}$ However, the advantages of OPCAB over ONCAB remain controversial. ${ }^{22)}$ The ongoing Coronary Artery Bypass Grafting Surgery Off- or On-Pump Revascularization Study trial (CORONARY trial) enrolled 4752 patients to investigate short- and long-term outcomes, including comorbidities after either OPCAB or ONCAB. This trial failed to prove the superiority of $\mathrm{OPCAB}$ over $\mathrm{ONCAB}$, but its inferiority was not shown as well. ${ }^{20,23)}$ Our present report also showed acceptable incidences of adverse events during the early postoperative period. Sufficiently low mortality and morbidity rates after OPCAB, as shown in this report, have encouraged us to promote OPCAB even more actively in the recent years.

Furthermore, we have recently performed LAA A/C simultaneously with OPCAB. The reason why we have added this procedure to $\mathrm{OPCAB}$ is because we assume that an add-on LAA procedure may be effective for the prevention of postoperative stroke or other systemic embolisms. The LAA is the main source of emboli in patients with 
Table 6 Preoperative factors associated with mortality and combined morbidity in the overall cohort (data limited)

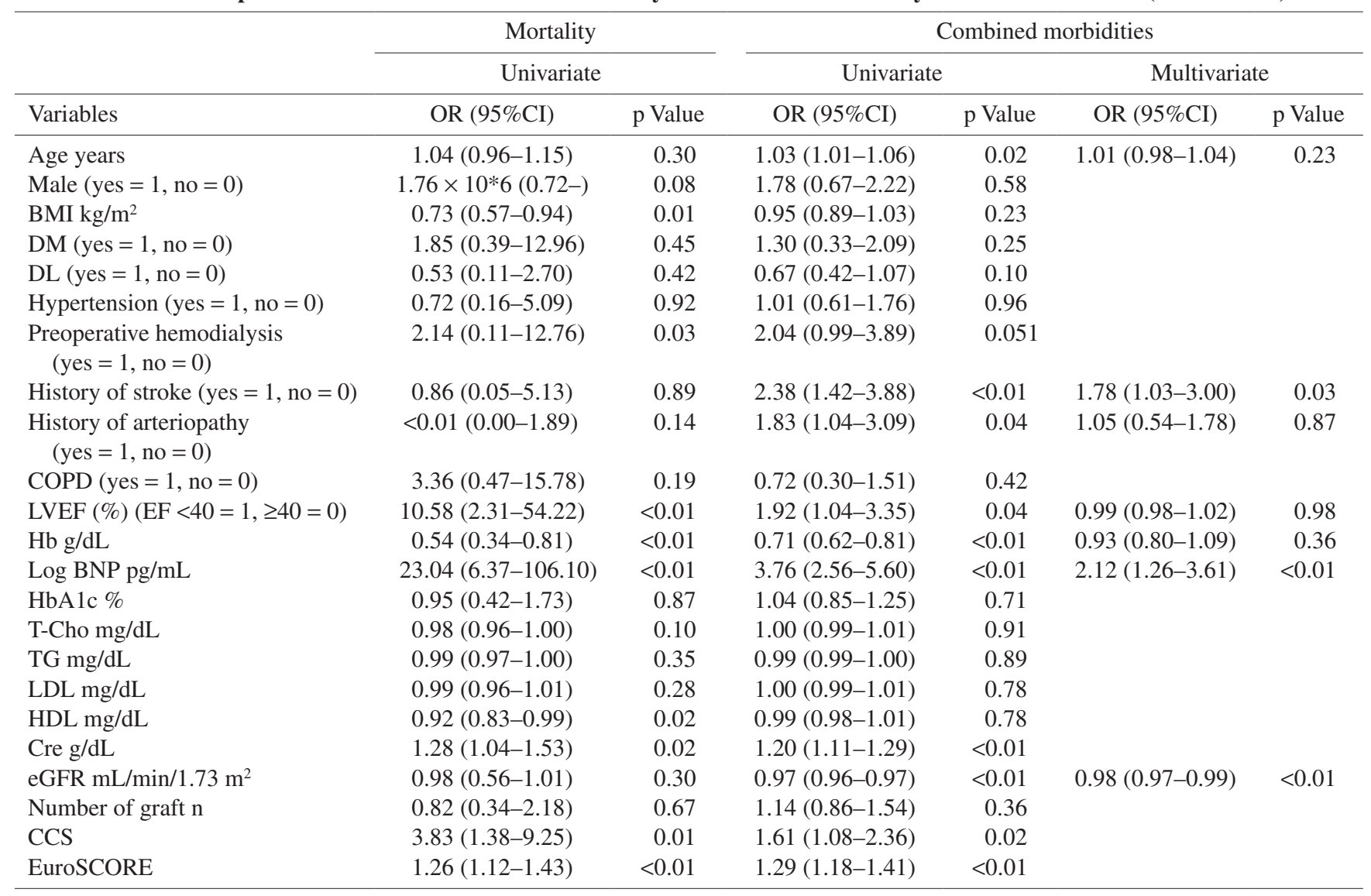

OR: odds ratio; CI: confidential interval; BMI: body mass index; DM: diabetes mellitus; COPD: chronic obstructive pulmonary disease; EF: ejection fraction; LVEF: left ventricular ejection fraction; BNP: brain natriuretic peptide; TG: triglyceride; LDL: low-density lipoprotein; HDL: high-density lipoprotein; eGFR: estimated glomerular filtration rate; CCS: Canadian Cardiovascular Society Functional Classification

Af. ${ }^{24)}$ Indeed, Af is the most prevalent arrhythmia encountered after cardiac surgery, and previous studies indicated that $20 \%-50 \%$ of patients develop postoperative Af. ${ }^{25)}$ Although the incidence of postoperative Af was reported to be lower in OPCAB patients than ONCAB patients, sizable patients definitely develop transient Af regardless of the type of procedure. Therefore, LAA A/C may be useful in preventing emboli-associated devastating adverse events even in patients undergoing OPCAB. Percutaneous LAA closure devices are now widely used especially in Europe. ${ }^{24)}$ However, such devices are not available in Japan. Hence, we initiated prophylactic LAA A/C simultaneously with $\mathrm{OPCAB}$, the results of which are now under prospective observational study. In addition, use of the aorta no-touch technique, in which in situ arterial grafts are used for all grafts to avoid manipulation of the ascending aorta, has been shown to reduce the occurrence of stroke. ${ }^{15)}$ Our institution also chose in situ arterial grafts whenever possible. The use of LAA A/C and the aorta no-touch technique might contribute to reducing perioperative mortality and morbidity rates.

Patients who undergo unplanned $\mathrm{ONCAB}$ as a result of intraoperative conversion from $\mathrm{OPCAB}$ have significantly higher rates of postoperative complications, operative mortality, and 30-day readmission. ${ }^{26)}$ The mean duration of postoperative hospital stay in OPCAB patients at our institution was 11 days, whereas those of ONCAB patients (elective and urgent) and a subset of five patients with urgent conversion from $\mathrm{OPCAB}$ to $\mathrm{ONCAB}$ were 22 and 26 days, respectively. We speculate that the prolonged hospitalization of the ONCAB patients may be related to their tough postoperative courses with several complications. In our institution, we select OPCAB rather than ONCAB for as many cases as possible, except for those with extremely poor preoperative left ventricular function. Unexpected CPB requirement occurred in patients whose PA pressure was elevated during apical elevation. These findings indicate that hemodynamic stability is one of the 
most important factors to accomplish OPCAB procedures in a successful manner. ${ }^{26,27)}$

Although an analysis identifying factor associated with mortality and morbidity based on our data contains statistical limitations, only low EF was found to be a possible predictive factor for a poor outcome as was shown in the previous reports. ${ }^{28)}$ Our results indicate that even under OPCAB procedures, patients with reduced systolic function may require special attention during their perioperative management in order to treat them as promptly as possible in case of adverse event occurrence.

The present paper is a preliminary report of more than 7 years of experience with OPCAB at one of the highestvolume centers in Japan. We admit that this is a cohort study; however, considering the fact that we perform $\mathrm{OPCAB}$ in a routine manner and $\mathrm{CPB}$ usage is an extreme exception at our institution, we believe our results are worth reporting. Due to the small number of ONCAB cases, we were unable to compare outcomes between $\mathrm{OPCAB}$ and $\mathrm{ONCAB}$. However, we believe that the clinical review of the patients' characteristics and early postoperative courses of the OPCAB patients in the present paper can provide useful information for other institutions that will engage in surgical coronary revascularization.

\section{References}

1) Lloyd-Jones D, Adams RJ, Brown TM, et al. Executive summary: heart disease and stroke statistics-2010 update: a report from the American Heart Association. Circulation 2010; 121: 948-54.

2) Nichols M, Townsend N, Scarborough P, et al. Cardiovascular disease in Europe 2014: epidemiological update. Eur Heart J 2014; 35: 2950-9.

3) Alderman EL, Kip KE, Whitlow PL, et al. Native coronary disease progression exceeds failed revascularization as cause of angina after five years in the Bypass Angioplasty Revascularization Investigation (BARI). J Am Coll Cardiol 2004; 44: 766-74.

4) Japanese Associate for Coronary Artery Surgery (JACAS). Coronary artery surgery results 2013, in Japan. Ann Thorac Cardiovasc Surg 2014; 20: 332-4.

5) Biglioli P, Cannata A, Alamanni F, et al. Biological effects of off-pump vs. on-pump coronary artery surgery: focus on inflammation, hemostasis and oxidative stress. Eur J Cardiothorac Surg 2003; 24: 260-9.

6) Milojević P, Nesković V, Stojanović D, et al. Offpump myocardial revascularization using the Octopus tissue stabilizer system. Med Pregl 2003; 56: 80-4.

7) Suzuki T, Asai T. Early and midterm results of off-pump coronary artery bypass grafting without patient selection. Heart Surg Forum 2008; 11: 213-7.
8) Kajimoto K, Yamamoto T, Amano A. Is off-pump CABG really inferior to on-pump strategies for longterm survival? J Am Coll Cardiol 2014; 64: 1181.

9) Bakaeen FG, Shroyer AL, Gammie JS, et al. Trends in use of off-pump coronary artery bypass grafting: Results from the Society of Thoracic Surgeons Adult Cardiac Surgery Database. J Thorac Cardiovasc Surg 2014; 148: 856-3.

10) Polomsky M, Puskas JD. Off-pump coronary artery bypass grafting - the current state. Circ J 2012; 76: 784-90.

11) Puskas JD, Thourani VH, Kilgo P, et al. Off-pump coronary artery bypass disproportionately benefits highrisk patients. Ann Thorac Surg 2009; 88: 1142-7.

12) Edgerton JR, Herbert MA, Jones KK, et al. On-Pump Beating Heart Surgery Offers an Alternative for Unstable Patients Undergoing Coronary Artery Bypass Grafting. Heart Surg Forum 2004; 7: 8-15.

13) Reber D, Brouwer R, Buchwald D, et al. Beating-heart coronary artery bypass grafting with miniaturized cardiopulmonary bypass results in a more complete revascularization when compared to off-pump grafting. Artif Organs 2010; 34: 179-84.

14) Tarakji KG, Sabik JF, Bhudia SK, et al. Temporal onset, risk factors, and outcomes associated with stroke after coronary artery bypass grafting. JAMA 2011; 305: 381-90.

15) Kajimoto K. Current State-of-the-art of coronary artery bypass surgery. J Japan Coron Assoc 2014; 4: 295-303

16) Kato TS, Iwamura T, Endo D, et al. Left atrial appendage closure reduces the incidence of postoperative cerebrovascular accident in patients undergoing cardiac surgery. Circ J 2015.

17) Peterson ED, Coombs LP, DeLong ER, et al. Procedural volume as a marker of quality for CABG surgery. JAMA 2004; 291: 195-201.

18) Shroyer AL, Grover FL, Hattler B, et al. On-pump versus off-pump coronary-artery bypass surgery. $\mathrm{N}$ Engl J Med 2009; 361: 1827-37.

19) Kuss O, von Salviati B, Börgermann J. Off-pump versus on-pump coronary artery bypass grafting: a systematic review and meta-analysis of propensity score analyses. J Thorac Cardiovasc Surg 2010; 140: 829-35.

20) Marui A, Okabayashi H, Komiya T, et al. Benefits of off-pump coronary artery bypass grafting in high-risk patients. Circulation 2012; 126: 151-7.

21) Møller CH, Perko MJ, Lund JT, et al. No major differences in 30-day outcomes in high-risk patients randomized to off-pump versus on-pump coronary bypass surgery: the best bypass surgery trial. Circulation 2010; 121: 498-504.

22) Lamy A, Devereaux PJ, Prabhakaran D, et al. Off-pump or on-pump coronary-artery bypass grafting at 30 days. N Engl J Med 2012; 366: 1489-97.

23) Lamy A, Devereaux PJ, Prabhakaran D, et al. Effects of off-pump and on-pump coronary-artery bypass grafting at 1 year. N Engl J Med 2013; 368: 1179-88. 
24) Reddy VY, Doshi SK, Sievert H, et al. Percutaneous left atrial appendage closure for stroke prophylaxis in patients with atrial fibrillation: 2.3-Year Follow-up of the PROTECT AF (Watchman Left Atrial Appendage System for Embolic Protection in Patients with Atrial Fibrillation) Trial. Circulation 2013; 127: 720-9.

25) Yadava M, Hughey AB, Crawford TC. Postoperative atrial fibrillation: incidence, mechanisms, and clinical correlates. Cardiol Clin 2014; 32: 627-36.

26) Li Z, Amsterdam EA, Danielsen B, et al. Intraoperative conversion from off-pump to on-pump coronary artery bypass is associated with increased 30-day hospital readmission. Ann Thorac Surg 2014; 98: 16-22.

27) Chowdhury R, White D, Kilgo P, et al. Risk factors for conversion to cardiopulmonary bypass during offpump coronary artery bypass surgery. Ann Thorac Surg 2012; 93: 1936-41.

28) Keeling WB, Williams ML, Slaughter MS, et al. Offpump and on-pump coronary revascularization in patients with low ejection fraction: a report from the society of thoracic surgeons national database. Ann Thorac Surg 2013; 96: 83-8. 